\title{
Factores Facilitadores de la Violencia Laboral: Una Revisión de la Evidencia Científica en América Latina
}

\section{ENABLING FACTORS OF WORKPLACE VIOLENCE: A REVIEW OF THE SCIENTIFIC EVIDENCE IN LATIN AMERICA}

\author{
Juan Pablo Toro', Constanza Gómez-Rubio² \\ 1. Programa de Estudios Psicosociales del Trabajo, Universidad Diego Portales, Santiago, Chile. \\ 2. Universidad Autónoma de Barcelona, Barcelona, España.
}

\section{RESUMEN}

En el presente artículo se dan a conocer los resultados de una revisión sistemática de la literatura que reporta investigaciones en poblaciones latinoamericanas en torno a factores facilitadores de la violencia laboral. Para esto se realizó una búsqueda en las bases de Academic Search Complete (EBSCOhost), Fuente Académica Premier, PSICODOC, Scielo.org, JSTOR y SCOPUS. Se contemplaron estudios empíricos e indexados entre los años 2009 y 2014. Se trabajó con una selección de 17 artículos y se generaron cuatro categorías de análisis cualitativo: 1. Aproximación metodológica de los estudios. 2. Factores determinantes individuales. 3. Factores determinantes sociales-interpersonales. 4. Factores determinantes organizacionales. A partir del análisis se concluye que la violencia en el trabajo tiene un carácter multideterminado, transversal y multicausal, por lo cual debe existir una perspectiva sistémica para su abordaje, con el fin de abarcar toda su complejidad y profundidad.

(Toro J, Gómez-Rubio C, 2016. Factores Facilitadores de la Violencia Laboral: Una Revisión de la Evidencia Científica en América Latina. Cienc Trab. May-Ago; 18 [56]: 110-116).

Palabras clave: VIOLENCIA LABORAL, REVISIÓN DE LITERATURA, ORGANIZACIONES, TRABAJO.

\section{ABSTRACT}

This article discloses the results of a systematic review of the literature reporting research in Latin American populations around facilitating factors of workplace violence. For this, a search was conducted on the bases of Academic Search Complete (EBSCOhost) Academic Source Premier, PSICODOC, Scielo.org, JSTOR and Scopus. Empirical studies were contemplated and indexed between 2009 and 2014. We worked with a selection of 17 articles, and four categories of qualitative analysis were generated: 1. Methodological approach of the studies. 2. Individual determinants. 3. social-interpersonal determinants. 4. organizational determinants. The analysis concluded that violence at work has a multidetermined, transversal and multi-causal character, so there must be a systemic perspective to address them, in order to cover all its complexity and depth.

Keywords: WORKPLACE VIOLENCE, LITERATURE REVIEW, ORGANIZATIONS, WORK.

\section{INTRODUCCIÓN}

Conforme las organizaciones hacen esfuerzos para adaptarse a contextos de continuos cambios de orden económico, tecnológico y social, en el último tiempo se ha evidenciado un importante crecimiento de la violencia en el trabajo. ${ }^{1}$

El interés de organismos públicos e investigadores en el tema se ilustra por la gran cantidad de literatura de diverso tipo que viene produciéndose en las últimas dos décadas. Un ejemplo de esa literatura y un aporte relevante y fundacional para la divulgación

Correspondencia / Correspondence:

Juan Pablo Toro

Vergara 275, Santiago, Chile

Tel.: +56 226762501

e-mail: juan.toro@udp.cl

Recibido: 02 de Febrero 2016 / Aceptado: 09 de Julio de 2016

y posicionamiento del problema de la violencia en el trabajo lo constituye el texto de Chapell y Di Martino ${ }^{2}$, originalmente publicado en 1998, que ofrece una revisión amplia y global del tema aportando a la comprensión del fenómeno, a la sistematización de las formas en que se ha confrontado y a las propuestas de acción hacia el futuro.

La violencia en el trabajo ha sido definida por la OIT $^{3}$ como "toda acción, incidente o comportamiento que se aparta de lo razonable mediante el cual una persona es agredida, amenazada, humillada o lesionada por otra en el ejercicio de su actividad profesional o como consecuencia directa de la misma" (p. 4). Se entiende, entonces, que la gama de acciones o incidentes violentos puede ser muy amplia, desde las manifestaciones abiertas de violencia física o verbal hasta las formas más sutiles, pero no por ello menos graves, como el acoso, ya sea psicológico o sexual y las manifestaciones de incivismo, aún más difícilmente catalogadas como formas de violencia en el lugar de trabajo.

El amplio espectro de comportamientos que cubre la violencia en el trabajo ha hecho necesario categorizarla en distintos tipos. Una forma de ordenamiento que ha tenido aceptación en la literatura 
especializada es la que ofrece la California Division of Occupational Health and Safety (Cal/OSHA). ${ }^{4}$ Originalmente planteada como una forma de sistematizar las expresiones más abiertas de violencia, aquellas que implican riesgo para la vida o daño físico, esta categorización también reconoce e incorpora las formas de violencia más sutil, y utiliza como criterio el origen de quien perpetra el comportamiento violento. Diferencia tres tipos de violencia: Tipo I: provocada por agentes externos sin vínculo legitimo con la víctima; Tipo II: provocada por usuarios, clientes, pacientes, consumidores; Tipo III: provocada por colegas, subalternos o jefes.

La propuesta de este estudio es inclusiva, esto es, pretendemos seguir los lineamientos señalados por Anderson y Pearson ${ }^{5}$ y luego por Cortina y Magley ${ }^{6}$, en cuanto a que las formas de violencia laboral constituyen una espiral que incluye muy diversas forma de expresión, por una parte, $y$, por otra, nos parece sugerente la propuesta de Hershcovis ${ }^{7}$ respecto a la conveniencia de utilizar un término genérico para aludir a estas conductas -él sugiere violencia en el puesto de trabajo (workplace aggression)-, ya que las distinciones que hace la literatura entre distintos términos en uso parecen no tener suficiente respaldo como para hacer de ellos constructos claramente diferentes, lo que podría ser incluso un obstáculo a la generación de conocimiento en el área. En este marco, el estudio de los antecedentes y facilitadores de la violencia laboral es reciente y se ha realizado desde una perspectiva "accidental", vale decir, a partir de causas de situaciones imprevistas. No obstante, el acoso laboral, que constituye la expresión de violencia laboral que ha focalizado el interés de los investigadores, muchas veces se establece como un fenómeno predeterminado y condicionado desde el inicio por factores de distinta índole que dan lugar a expresiones sutiles de violencia o maltrato que afirman y dan sustento al carácter multideterminado y procesual del fenómeno. ${ }^{8}$ Así como son diversos sus determinantes, las distinciones entre formas de presentación, intensidad y efectos de los comportamientos violentos no permiten establecer con claridad límites precisos que permitan identificar si se trata de acoso, violencia ocasional, o maltrato generalizado.

Siguiendo el análisis de la literatura escandinava que hace Einarsen en relación al acoso laboral ${ }^{9}$, la explicación de los procesos de violencia laboral puede ser conceptualizada bajo tres formas: 1. En relación a las variables individuales de personalidad, tanto de acosadores y de víctimas; 2. En función de las características inherentes a las relaciones interpersonales; 3. A partir de las características del entorno socio-laboral. El primero de los enfoques plantea que existen ciertas características de vulnerabilidad en los/as trabajadores/as que sufren de acoso laboral. El segundo, responde a una mirada antropológica, en la que los conflictos interpersonales serían propios de las relaciones sociales y, por tanto, esperables. La tercera perspectiva postula que un entorno laboral débilmente organizado propicia la aparición de situaciones de violencia. Un análisis preliminar de la literatura permite afirmar que son los factores organizacionales los que han tenido mayor aceptación en la explicación del mobbing.8 Abundante evidencia señala la importancia de los factores del contexto organizacional en la facilitación de la emergencia del acoso, identificando distintos aspecto de la organización del trabajo, de las formas de gestión, los valores y la cultura organizacional como elementos relevantes. ${ }^{10-13}$ Algunos autores destacan como determinantes los entornos de trabajo estresantes; lugares en donde es altamente valorada la competencia; contextos laborales en que se niega la existencia de mobbing; y, donde hay una ausencia de ética laboral en la organización. ${ }^{8}$ Adicionalmente, para Tobin $^{1}$, la estructura organizacional tiene un efecto en la generación de violencia en el lugar de trabajo; así, aquellas organizaciones con estructuras burócratas, jerárquicas, rígidas y autoritarias, serían más proclives a sufrir violencia laboral. ${ }^{14}$

Por otro lado, Moreno et $\mathrm{al}^{15}$ evidencian que la política organizacional, el tipo de contrato y la actuación en torno a la violencia predicen de forma significativa la aparición de ésta en las organizaciones, siendo la primera la que tiene mayor capacidad predictiva.

También se ha señalado que los empleos que viven constantemente cambios en sus sistemas productivos y que, por tal, van transformándose en el tiempo y aumentando la inestabilidad, incrementan la posibilidad de aparición de acoso laboral. ${ }^{16} \mathrm{~A}$ su vez, los contratos temporales se han asociado al aumento de la indefensión ante situaciones de abuso psicológico, físico y verbal en el contexto de trabajo. ${ }^{15}$

Los estudios que abordan la violencia laboral desde la perspectiva de las relaciones interpersonales asumen que la violencia laboral es parte de la vida cotidiana de las organizaciones y que el acoso (y la violencia en general, agregamos nosotros) sería una característica inherente a las relaciones sociales. ${ }^{17}$

Por otra parte, los estudios que se focalizan en las características de personalidad de las víctimas han identificado, entre otros factores, baja autoestima y ansiedad social ${ }^{18} \mathrm{o}$ altas expectativas de logro no ajustadas a sus capacidades y recursos ${ }^{17}$, aunque un problema de esta línea de estudios es que, al no tratarse de diseños longitudinales, los rasgos de personalidad pueden ser tanto causa como consecuencia de la victimización.

Un meta-análisis ${ }^{13}$ agrupa en dos grandes categorías los modelos que respaldan la investigación en torno al acoso laboral: una categoría releva las características demográficas o de personalidad de las víctimas; y, la otra, destaca las condiciones del ambiente de trabajo como predictores. En este sentido, los estudios que enfatizan las características individuales de las víctimas (sociodemográficas o de personalidad) identifican a los jóvenes y a las mujeres como víctimas frecuentes del acoso, e identifican como características personales, entre otras, la baja autoestima y la elevada ansiedad. Por su parte, los estudios focalizados en características organizacionales identifican como factores asociados al acoso la mayor ambigüedad, el conflicto de rol y la sobrecarga de tareas. Otras condiciones de la organización operan como filtro frente al acoso laboral, tales como la orientación a la justicia organizacional; mientras que el predominio de valores tales como la competitividad, eficacia y rendimiento se asocia a un acoso más frecuente y tolerado. ${ }^{13}$

Esta misma distinción se mantiene en la literatura actual cuando se trata de diferenciar o categorizar la gran variedad de factores causales de la violencia en el trabajo. Así, por ejemplo, más recientemente, Samnani y Singh ${ }^{19}$, al hacer una revisión de 20 años de investigación en torno al bullying, identifican cuatro niveles de antecedentes y consecuencias: individual, grupal, organizacional y societal.

El presente artículo se enmarca en el proyecto Dimensiones Organizacionales de la Violencia en el Trabajo en Chile del Fondo Nacional de Desarrollo Científico y Tecnológico de Chile, y tiene como objetivo informar los resultados de una revisión sistemática de la literatura que reporta investigaciones en poblaciones latinoamericanas en torno a factores facilitadores de la violencia laboral. 


\section{OBJETIVOS}

En el marco de un creciente interés por la investigación sobre el tema en la literatura latinoamericana ${ }^{20}$, el objetivo de este trabajo es identificar los factores determinantes o facilitadores de la violencia laboral que han sido objeto de estudio en el marco de la literatura científica de la región y someter a análisis estos estudios de modo de lograr identificar sus características distintivas. Para este análisis utilizaremos el modelo propuesto por Einarsen ${ }^{9}$, identificando factores facilitadores de la violencia laboral de los tres órdenes descritos que, como se ha dicho, corresponden a grandes categorias que la literatura especializada ha utilizado.

\section{MÉTODO}

El presente estudio es de carácter descriptivo y exploratorio. Por una parte, busca recolectar información que muestre aspectos y características del fenómeno estudiado y, por otra, examina un problema poco estudiado y del cual no existe mucha información. ${ }^{21}$

Se llevó a cabo una revisión sistemática cualitativa de la literatura en el ámbito latinoamericano utilizando técnicas cualitativas para establecer categorías y subcategorías de análisis. Las revisiones sistemáticas son investigaciones cientificas que utilizan como unidad de análisis fuentes de información primarias. Así, implican la búsqueda exhaustiva de todos los estudios relevantes al objetivo de investigación, y con criterios explícitos y reproducibles. ${ }^{22}$

Se utilizó Análisis de Contenido Cualitativo -(ACC)- para el análisis de la información, entendiéndolo como un análisis controlado del proceso comunicativo entre el texto y el contexto, que debe responder a las reglas para su proceder. El procedimiento permite dividir los textos en unidades de análisis, lo que facilita su estudio y da un mayor acercamiento y conocimiento de los textos. Además, permite la generación de categorías analíticas que fomentan la construcción de ideas fundamentales. En términos de validez, el ACC otorga la posibilidad de triangulación de los resultados con estudios similares. ${ }^{23}$

La búsqueda de la literatura se realizó utilizando las siguientes bases de datos: Academic Search Complete, Fuente Académica Premier, PSICODOC, Scielo.org, JSTOR y SCOPUS.

Los criterios de inclusión fueron: 1. Estudios cualitativos y cuantitativos que hicieran referencia a la violencia. 2. Estudios empíricos realizados con población latinoamericana. 3. Estudios publicados entre los años 2009 y 2014; vale decir, se utilizó el criterio utilizado por la comunidad cientifica respecto a la validez de las investigaciones por un período de los últimos 5 años. ${ }^{24} 4$. Literatura escrita en español, inglés y portugués. 5. Ser artículos publicados en revistas científicas indexadas en ISI, SCOPUS, Scielo y Latindex, con el fin de asegurar la calidad de las publicaciones.

Los términos de búsqueda o palabras claves en español fueron: "violencia laboral", "Acoso laboral", "Acoso psicológico + trabajo" y "Acoso moral". En inglés fueron: "Mobbing", "Harassment + work", "Workplace bullying” y "Work violence". Por último, los términos en portugués: "Violência no Trabalho", "Assédio no trabalho", "Assédio moral + trabalho", "Mobbing”, "Assédio moral", "Assédio + trabalho", "Assédio moral no trabalho". Como resultado del procedimiento señalado, se obtuvieron 46 artículos generales, de los cuales se escogieron 17 trabajos que hacian referencia, específicamente, a los factores determinantes o facilitadores de la violencia laboral.

\section{PROCEDIMIENTO DE ANÁLISIS}

Según Ferrerira, Urrútia, y Alonso-Coello ${ }^{22}$, las revisiones sistemáticas siguen el siguiente orden:

1. Definición de la pregunta y objetivo de estudio. Asimismo, se plantean los criterios de inclusión y exclusión muestral.

2. Selección de la literatura relevante.

3. Extracción de los datos desde las fuentes primarias.

4. Análisis de los resultados.

5. Interpretación de los resultados.

En consonancia con lo anterior, en el presente estudio se trabajó y organizó el material de la siguiente manera:

1. Todos los trabajos seleccionados según los criterios preestablecidos fueron leídos e ingresados a una "Matriz de registro", utilizando una base de datos Excel. La matriz consideró los elementos descritos en la Tabla 1.

2. Se procedió a identificar categorías y subcategorías de análisis, según la "Matriz de registro". En un comienzo, el foco se colocó en el contenido manifiesto de los textos, y luego en los contenidos latentes de los mismos.

3. Una vez analizado el contenido de la literatura, se procedió a estudiar las metodologías empleadas en los estudios. Para esto, el enfoque fue identificar qué tipo de metodología se usaba y qué técnicas de recolección de datos eran utilizadas.

4. Los resultados fueron discutidos e interpretados.

5. Finalmente, el análisis y resultados fueron redactados.

\section{RESULTADOS}

Caracterización de los estudios seleccionados:

Tal como es posible apreciar en la Tabla 2, se seleccionaron 17 trabajos empíricos.

De los artículos seleccionados, 14 (82\%) corresponden a investigaciones llevadas a cabo con muestras de población de Brasil, uno de

Tabla 1.

Definición de categorias de análisis.

\begin{tabular}{|c|c|}
\hline Nombre de la categoría & Definición \\
\hline Año & Fecha de la publicación. \\
\hline Revista & Revista de origen de la publicación del artículo \\
\hline Pais & Pais de origen muestral. \\
\hline Metodología & Estudio cualitativo, cuantitativo o mixto. \\
\hline Número de muestra & Número de la población con la que se trabajó. \\
\hline Conductas que expresan Violencia & $\begin{array}{l}\text { Manifestaciones que dan cuenta de algún tipo } \\
\text { de violencia laboral. }\end{array}$ \\
\hline Factores Facilitadores de Violencia & $\begin{array}{l}\text { Condiciones contextuales que fomentan la } \\
\text { aparición del fenómeno de la violencia. }\end{array}$ \\
\hline Impactos en la salud & $\begin{array}{l}\text { Efectos físicos, psicológicos o conductuales que } \\
\text { se producen por la violencia laboral }\end{array}$ \\
\hline $\begin{array}{l}\text { Mecanismos individuales } \\
\text { de afrontamiento }\end{array}$ & $\begin{array}{l}\text { Estrategias conductuales o psicológicas que } \\
\text { despliegan las víctimas de violencia laboral. }\end{array}$ \\
\hline $\begin{array}{l}\text { Mecanismos organizacionales de } \\
\text { afrontamiento }\end{array}$ & $\begin{array}{l}\text { Medidas que ponen en marcha las instituciones } \\
\text { cuando se produce violencia laboral. }\end{array}$ \\
\hline Población trabajadora más afectada & $\begin{array}{l}\text { Población o individuos que son potencialmente } \\
\text { afectados por violencia laboral. }\end{array}$ \\
\hline Sector ocupacional & Señalar el sector en el cual se realizó el estudio \\
\hline Tipo de Trabajadores & Define la jerarquia ocupacional de los trabajadores \\
\hline Tipo de Violencia & $\begin{array}{l}\text { Indicar si corresponde a física, psicológica, } \\
\text { sexual o mixta. }\end{array}$ \\
\hline
\end{tabular}

Fuente: Elaboración propia. 
Tabla 2.

Artículos seleccionados.

\begin{tabular}{|c|c|c|c|}
\hline Autores & Año & Nombre de publicación & Revista \\
\hline Lima, C., et al. & 2014 & $\begin{array}{l}\text { Assédio moral e violências no trabalho: caracterização empericia judicial. } \\
\text { Relato de experiência no setor bancário }\end{array}$ & Revista Brasileira de Saúde Ocupacional \\
\hline Caran, V.C.S. et al., & 2010 & Assédio moral entre docentes de instituição pública de ensino superior do Brasil & Acta Paulista de Enfermagem \\
\hline Marques, R. C. et al. & 2012 & Assédio Moral nas Residências Médica e Não Médica de um Hospital de Ensino & Revista brasileña de educacion medica \\
\hline Jacoby, A. R., et al & 2009 & Assédio moral: uma guerra invisible no contexto empresarial & Revista Malestar e Subjetividade \\
\hline Jacoby, A.R. y Monterio, J. K. & 2014 & Mobbing of Working Students & Paidéia \\
\hline Antunes $B$, et al & 2012 & Mulher e trabalho: visibilizando o tecido e a trama que engendram o assédio moral & Psicologia em Revista \\
\hline Soares, L.R. and Villela, W. V. & 2012 & 0 assédio moral na perspectiva de bancários & Rev. bras. Saúde Ocup. \\
\hline Rigotto $R_{\text {, et al }}$ & 2010 & $\begin{array}{l}\text { Produtividade, pressão e humilhação no trabalho: os trabalhadores } \\
\text { e as novas fábricas de calçados no Ceará }\end{array}$ & Rev. bras. Saúde Ocup. \\
\hline Vasconcellos, I.R.R. et al. & 2012 & Violence in daily hospital nursing work & Acta Paulista de Enfermagem \\
\hline Batista, C. B. et al. & 2011 & $\begin{array}{l}\text { Violência no trabalho em saúde: análise em unidades básicas de saúde } \\
\text { de Belo Horizonte, Minasgerais }\end{array}$ & Trab. Educ. Saúde \\
\hline Escartín J. et al & 2011 & Workers' perception of workplace bullying: A cross-cultural study & $\begin{array}{l}\text { European Journal of Work and } \\
\text { Organizational Psychology }\end{array}$ \\
\hline Souza Junior, A. et al., & 2013 & $\begin{array}{l}\text { Aspectos da dominação masculina no assédio moral ao profissional } \\
\text { homossexual no polo industrial de manaus. }\end{array}$ & Revista Pensamento \& Realidade \\
\hline
\end{tabular}

Fuente: Elaboración propia.

Argentina, uno de México y otro es de carácter transcultural, que incluye muestras de Costa Rica y España. Sólo tres de los trabajos $(17,6 \%)$ no tratan el tema del acoso psicológico, sino se focalizan en la violencia laboral general. En cuanto al tipo de factores facilitadores que estos estudios refieren, 12 de ellos (70,5\%) identifican factores de la organización, 4 (23,5\%) identifican factores socialesinterpersonales y $5(29,4 \%)$ identifican variables individuales. Cabe destacar que 3 estudios fueron clasificados en dos categorías.

Resulta relevante destacar que, si bien se logró asignar los trabajos a las categorias previstas según el énfasis o foco principal de su problematización y objetivos, la mayoría de ellos las cruzan transversalmente, en espacial al momento de discutir sus resultados. De esta manera, aun cuando el estudio tenga un foco individual, el contexto social y organizacional no pasa inadvertido y es tema de reflexión, lo que parece señalar que se está concibiendo la violencia laboral y, en particular, el acoso psicológico, como un fenómeno multideterminado cruzado por variables de distinto tipo. Así, por ejemplo, Fontes et $\mathrm{al}^{26}$ afirman que su artículo sobre determinantes del bullying en enfermeras, se focaliza en determinantes de tipo individual "is only the first step to evaluate other factors of influence related to the organizational context" (p. 758). Del mismo modo, Rodríguez ${ }^{27}$ reconoce en el contexto académico la existencia de atmósferas psicosociales tóxicas generadas por algunos jefes y califica el entorno, donde sitúa su estudio, como generador de estrés por su estructura altamente burocratizada y expuesta a arbitrariedades, donde se facilita la aparición del mobbing. Otros autores son más explícitos para destacar el carácter extra-individual del acoso psicológico, como lo hacen Soares y Villela ${ }^{28}$ cuando anotan "...é necessário tratar o assédio moral em uma perspectiva coletiva, como uma questão social, e não individual" (p. 203), entendiendo que el estudio de este fenómeno no debe limitarse a la subjetividad de sus protagonistas, sino abarcar también al sistema sociopolítico que engendra este tipo de conflicto.

El abordaje de la mayoría de los estudios incorpora explícitamente como objetivo el acoso laboral, siendo diversas las poblaciones estudiadas (personal de salud, trabajadores de servicios e industriales). De los tres trabajos que se alejan de esta línea y abordan la violencia laboral desde una perspectiva más genérica, uno de ellos se propone indagar la violencia laboral considerándola en un sentido más amplio, para lo cual la describe y clasifica como física, sexual o verbal ${ }^{29}$; otro se enfoca en las agresiones provocadas por usuarios de servicios de salud y manifestadas de diversas formas. ${ }^{30}$ El tercer artículo indaga en la humillación como una práctica de violencia que persigue objetivos de productividad. ${ }^{31}$

Caracterización de la aproximación metodológica de los estudios: En cuanto a las metodologías utilizadas por los trabajos revisados, una primera constatación es que nueve de ellos utilizan una aproximación metodológica cualitativa y ocho son de índole cuantitativa. Los instrumentos utilizados en los estudios cuantitativos son, en su mayoría, cuestionarios autoconstruidos en forma ad-hoc para los propósitos de los respectivos estudios, pero también se reporta la utilización de las dos versiones del LIPT (Leyman Inventory of Psychlogical Terrorization) 45 y 60, así como el NAQ (Negative Acts Questionnaire). ${ }^{18}$ Un estudio informa la aplicación de un instrumento propio que utilizó ítems del cuestionario CISNEROS. ${ }^{32}$ Llama la atención que no se reporte la utilización del inventario latinoamericano disponible, el IVAPT-Pando, instrumento cuyo uso se ha venido extendiendo por la región y que ha sido sometido a validaciones en distintos paises. ${ }^{3-37}$ 
De manera general, los estudios cualitativos declaran la importancia de conocer las experiencias de violencia en profundidad, a partir de los mismos relatos de los/as involucrado/as. Las investigaciones de esta línea han hecho uso principal del análisis de contenido de entrevistas semi-estructuradas individuales y grupos focales. Un estudio somete la transcripción de sus entrevistas a análisis del discurso, para buscar las formaciones discursivas de los significados construidos entre el sujeto y el contexto. En este sentido, el énfasis estuvo puesto en los pensamientos, sentimientos, valores y creencias de los participantes del estudio, en relación a la violencia. ${ }^{38}$ Un segundo estudio, también somete los datos a análisis de discurso, para colocar de relieve el valor político del lenguaje, para dar cuenta de ideologías, relaciones de poder, de sumisión y resistencia. ${ }^{39}$

\section{Los factores determinantes individuales}

En un estudio realizado en Brasil, con una muestra de 199 trabajadores de enfermería, se identifican como variables asociadas a la victimización el sexo femenino, tener hijos, trabajar en la institución durante un período de 1 a 3 años. ${ }^{40}$

Un estudio realizado en Argentina con una muestra de 300 académicos mostró, junto a otros factores de índole organizacional, que las víctimas de acoso diferían del resto del grupo en cuanto a valores, actitudes y comportamientos, sin mayor especificación. ${ }^{27}$ Por su parte, en un estudio realizado en Brasil con trabajadores diagnosticados con LER/DORT, se identifica como variable individual relevante, asociada a la victimización por acoso laboral, la poca disponibilidad de herramientas para enfrentar la conducta abusiva de la cual son objeto. ${ }^{41}$

Otro estudio realizado en Brasil, con una muestra de 175 empleados del sector de la educación superior, asoció los estilos personales de manejo de conflictos (cooperación-dominación) a los efectos protectores o de riesgo sobre el acoso psicológico. El estudio concluye que los estilos personales que combinan dominación (del victimario) y evitación (por parte de la víctima) constituyen un factor de riesgo para sufrir acoso moral en el trabajo. Por otra parte, se identifica el estilo cooperador como posible factor protector. $^{42}$

Un estudio cualitativo realizado con empresarios en Brasil $^{43}$ sugiere que las víctimas de acoso tienen un perfil psicológico fuerte, son competentes y destacadas en sus lugares de trabajo, a diferencia de los supuestos que hablan del carácter frágil de las víctimas.

\section{Los factores determinantes sociales-interpersonales}

El mismo estudio con víctimas de acoso en el ámbito empresarial identifica factores de contexto tales como el capitalismo y la competitividad del mundo moderno y la falta de conocimiento legal en los trabajadores. Por otro lado, los autores afirman que el acoso se facilita en organizaciones carentes de reglas precisas, jerárquicas. ${ }^{43}$ Por su parte, también en Brasil, Soares y Villela ${ }^{28}$ informan un estudio cualitativo con 37 trabajadores bancarios con quienes indagan su concepto del acoso laboral. En un ambiente laboral incierto y exigente, ellos reconocen como factores facilitadores de la violencia laboral los constantes cambios a los que se ven expuestos en términos de organización del trabajo. Otro estudio brasileño de índole cualitativa, esta vez con 17 docentes de la enseñanza superior privada ${ }^{44}$, identifica como factores asociados al acoso laboral el contexto moderno, el alto desempleo, el modelo laboral y económico incierto y la fuerte competitividad en este tipo de instituciones. Los empleados temen perder su trabajo y fracasar, por lo que se quedan en las empresas y aguantan toda clase de malos tratos.
Por último, una cuarta investigación, que alude a factores de tipo social, es la de Souza Junior et $\mathrm{al}^{39}$, realizado en Brasil. Con una metodología cualitativa sobre 6 casos, estudian el acoso moral y violencia en el trabajo en el contexto de discriminación sexual a homosexuales, e identifican como factor social determinante la dominación masculina y las desigualdades de género. Se evidenció que la cultura puede producir expectativas en el comportamiento de las personas que son transmitidas en diversas instituciones sociales, entre ellas el trabajo. En éste, las conductas contra trabajadores homosexuales ocurren de manera directa y verbal, lo que muchas veces se traduce en situaciones que los atemorizan cotidianamente, mediante diversas formas de exclusión y discriminación. El acoso moral hacia personas homosexuales en el lugar de trabajo se traducía en una forma de invisibilización que lleva a la negación de la existencia legitima, reconocida y pública.

\section{Los factores determinantes organizacionales}

De la revisión de los artículos que señalan factores del entorno sociolaboral y organizacional directa o indirectamente relacionados a distintas formas de violencia, tres de ellos aluden a dimensiones extra-organizacionales que se asocian a las nuevas formas de management, entendidas como la cultura laboral moderna, caracterizada por la búsqueda de la rapidez, la eficiencia y optimización ${ }^{45}$, al capitalismo actual y la competitividad del mundo moderno, en que se aprecia una falta de conocimiento legal de los trabajadores. ${ }^{43}$ En esta misma línea, Rodrigues y Freitas ${ }^{44}$ mencionan como factores facilitadores el alto desempleo, un modelo laboral y económico incierto y la alta competitividad entre las empresas, que tienen por consecuencia que los empleados teman perder su trabajo y fracasar, por lo que soportan diversas formas de maltrato.

En cuanto a características internas, propias de la organización específica más que del entorno social y cultural, se identifican factores tales como la falta de equidad y solidaridad de los ambientes altamente competitivos, así como las estructuras organizacionales burocráticas, autoritarias, jerárquicas y rígidas. ${ }^{46,47,27,41}$ Un artículo da cuenta de las características propias de las instituciones de salud, identificando como factores determinantes de la violencia el contacto directo con pacientes, las condiciones precarias del trabajo y la insuficiente cantidad de personal. ${ }^{29}$

También en el entorno de instituciones de salud, Batista et $\mathrm{al}^{30}$ se refieren en su artículo a la índole del trabajo de servicios que, al ser altamente demandante, opera como un predictor de violencia.

\section{DISCUSIÓN}

La violencia laboral ha sido reconocida como un problema de creciente relevancia social, por las graves consecuencias que produce. En este contexto, se ha evidenciado un aumento del interés por parte de la literatura latinoamericana en la temática. ${ }^{20}$ En virtud de ello y del objetivo del presente estudio, se pudieron conocer los factores determinantes o facilitadores de la violencia laboral que han sido estudiados por la literatura científica de la región. Así, un primer elemento a destacar es la clara preeminencia del acoso psicológico como tema central de los artículos (representa un 82\% de la muestra). Por cierto, esto era esperable a partir de las palabras clave de búsqueda, pero la inclusión entre esos términos de "violencia laboral" permitía esperar también que algunos de los artículos se abocaran a un tratamiento más amplio del fenómeno, tal como éste es cubierto en revisiones de carácter más general, como los hacen, por ejemplo, 
Chapell y Di Martino², en un texto que constituye una referencia indispensable en el tema. Llama la atención la concentración que constatamos en esta muestra de estudios latinoamericanos en uno de los fenómenos de violencia laboral, cuando la literatura general tiende a mostrar, por el contrario, una profusión de conceptos de difícil delimitación que puede resultar por momentos confusa.?

Si se considera la clasificación del tipo de violencia de acuerdo al perpetrador, según plantea $\mathrm{Cal} / \mathrm{OSHA}^{4}$, se constata que los trabajos analizados en esta revisión pertenecen en su gran mayoría a violencia tipo III. Esto es, se aprecia en ellos una preeminencia del interés por investigar en relación a la violencia ejercida por colegas y jefes y, particularmente, por el acoso psicológico, como se ha señalado. La violencia tipo II, ejercida por clientes o usuarios es mencionada sólo en un estudio en contexto sanitario, mientras la violencia provocada por agentes externos, sin vínculo legítimo con la víctima, no tiene presencia en los trabajos examinados.

En consonancia con la literatura general sobre violencia en el trabajo, que da cuenta de su carácter multideterminado, se constata en esta revisión que varios de los artículos analizados, aun cuando se focalizan en una de las tres categorias de factores determinantes utilizadas para el análisis, evidencian un alto grado de transversalidad al hacer referencia explícita y más o menos detallada a la multicausalidad del fenómeno, lo que, en algunos casos, hace difícil su asignación a una de las categorías analíticas.

Respecto a las metodologías empleadas por los estudios, mayoritariamente fueron de corte cualitativo, aunque la diferencia es de un artículo con respecto a la cuantitativa. La forma en que se estudia el fenómeno influye en la determinación de qué factores son relevantes de considerar en la investigación y publicación científica sobre violencia laboral. En este sentido, los estudios cuantitativos hacen uso de cuestionarios para investigar la violencia laboral, otorgando datos sobre factores demográficos que podrían influir en su aparición, como el de Fontes et $\mathrm{al}^{26}$, así también, los valores, actitudes, comportamientos y estilos de personalidad asociados al mismo, como los de Rodrígue ${ }^{27}$ y Acosta. ${ }^{42}$ De esta forma, fue posible apreciar que el estudio cuantitativo de los determinantes que facilitan la violencia en contextos laborales refieren, principalmente -aunque no exclusiva- a factores individuales.

Por otro lado, los artículos cualitativos colocan énfasis en el estudio de las subjetividades, las ideologías, y las relaciones asimétricas y de poder que se configuran como factores facilitadores de la violencia laboral. Estos tipos de estudios, como los de Meurer Antunes et $\mathrm{al}^{38}$; Souza Junior et $\mathrm{al}^{39}$; Soares y Villela ${ }^{28}$; Rodrigues y Freitas ${ }^{44}$, son transversales a los tres tipos de factores señalados por Einarsen ${ }^{9}$, aunque se marcan con mayor énfasis en los factores sociales-interpersonales y en los organizacionales.

En lo que refiere a los factores organizacionales de la violencia, lo encontrado en la revisión respecto a las características internas, tales como organizaciones altamente autoritarias, jerárquicas y condiciones precarias de trabajo, es concordante con lo planteado por Wlosko y Ros $^{16}$ y el Gobierno de Chile. ${ }^{14}$

\section{CONSIDERACIONES FINALES}

Tal como fue posible apreciar a lo largo de la revisión, los factores determinantes en la violencia laboral son variados, transversales y multidimensionales. En un intento analítico se han clasificado en tres tipos: individuales, sociales-interpersonales, y organizacionales; no obstante, en la práctica es difícil identificar procesos de violencia que respondan a un solo tipo e, incluso, muchas veces es complejo distinguir la violencia propiamente tal de ciertos fenómenos que habitualmente son conceptualizados de otras formas.

Ciertamente, no es posible analizar la violencia laboral desvinculada de otras problemáticas organizacionales, y menos aun de otros tipos de violencia social. En este sentido, la violencia debe ser vista como un problema sistémico, atravesado por aspectos micro y macro sociales, de relaciones cotidianas, pero también de la estructura del mercado laboral y de las formas de organización del trabajo que resultan de ella, las que van imponiendo un sistema cada vez más precarizado de condiciones y relaciones laborales. De este modo, no es posible reducir sus determinantes a características meramente individuales ni interaccionales, sino es necesario considerar la dimensión política del problema, recociendo la relevancia de los contextos que condicionan las relaciones humanas en los entornos organizacionales; de esta forma, se podrá comprender el fenómeno en toda su complejidad, permitiendo buscar soluciones que abarquen la profundidad del problema.

\section{REFERENCIAS}

1. Tobin T. Organizational determinants of violence in the workplace. Aggress Violent Beh. 2001:6:91-102.

2. Chapell D, Di Martino V. Violence at Work. Geneva: International Labour Office; 2006.

3. OIT. Repertorio de recomendaciones prácticas sobre violencia y el estrés en el sector servicios: una amenaza para la productividad y el trabajo decente. Ginebra: Oficina Internacional del Trabajo; 2003.

4. California, Department of Industrial Relations, Division of Occupational Safety and Health (DOSH), CAL/OSHA. Guidelines for workplace security [on line]. San Francisco;1995 [cited Jun 2016]. Disponible en: https://www.dir. ca.gov/dosh/dosh_publications/worksecurity.html

5. Anderson L M, Pearson CM. Tit for tat? The spiraling effect of incivility in the workplace. Acad Manage Rev.1999;24:452-471.

6. Cortina L, Magley V. Incivility in the workplace: incidence and Impact. J Occup Health Psych. 2001;6(1):64-80.

7. Hershcovis M.S. Incivility, social undermining, bullying. . .oh my!: A call to reconcile constructs within workplace aggression research. J Organ Behav.
$2011 ; 32: 499-519$.

8. González D, Delgado M. El acoso psicológico en el lugar de trabajo. Antecedentes organizacionales. Boletín de Psicologia (Valencia). 2008;93:7-20.

9. Einarsen E. Harassment and bullying at work: A review of the Scandinavian approach. Aggress Violent Beh. 2000; 5(4):379-401.

10. Di Martino V. Workplace violence in the health sector. Country case studies Brazil, Bulgaria, Lebanon, Portugal, South Africa, Thailand, plus an additional 14 Australian study; Synthesis Report [on line]. Geneva: ILO/ICN/WHO/PSI Joint Programme on Workplace Violence in the Health Sector; 2002 [cited Jun 2016]. Available from: http://www.who.int/violence_injury_prevention/injury/ en/WVsynthesisreport.pdf.

11. Organización Mundial de la Salud. Sensibilizando sobre el Acoso Psicológico en el Trabajo [en línea]. Ginebra: OMS; 2004 [citado jun 2016]. (Serie Protección de la Salud de los Trabajadores; 4). Disponible en: http://www. who.int/occupational_health/publications/harassment/es/

12. Piñuel I. Neomanagement. Jefes tóxicos y sus víctimas. Madrid: Aguilar; 2004. 
13. Topa G, Depolo M, Morales, JF. Acoso laboral: meta-análisis y modelo integrador de sus antecedentes y consecuencias. Psicothema. 2007;19(1):88-94.

14. Chile. Gobierno de Chile. El acoso laboral o mobbing [en línea]. Santiago: Dirección del Trabajo; 2007 [citado 20 ene 2016]: Disponible en: http://www. dt.gob.cl/m/1620/articles-95386_archivo_fuente.pdf

15. Moreno B, Rodríguez A, Garrosa E, Morante ME. Antecedentes organizacionales del acoso psicológico en el trabajo: un estudio exploratorio. Psicothema. 2005;17(4):627-632.

16. Wlosko $\mathrm{M}, \operatorname{Ros} \mathrm{C}$. Violencia laboral: hipótesis y niveles explicativos a la luz del caso del personal de enfermeria del sector público. I Congreso Internacional de Investigación y Práctica Profesional en Psicologia- XVI Jornadas de Investigación- Quinto Encuentro de Investigadores en Psicología del MERCOSUR; 6-8 ago 2009; Buenos Aires, Argentina. Buenos Aires; Universidad de Buenos Aires; 2009 [citado 20 ene 2016]. Disponible en: https://www.aacademica.org/000020/306.pdf

17. Brodsky C. The harassed worker. Lexington, MA: D.C. Health and Company; 1976.

18. Einarsen $\mathrm{S}$, Raknes $\mathrm{BI}$, Matthiesen SB. Bullying and harassment at work and their relationship to work environment quality: An exploratory study. Eur J Work Organ Psy. 1994; 4:381- 401

19. Samnani AK, Singh P. 20 Years of workplace bullying research: A review of the antecedents and consequences of bullying in the workplace. Aggress Violent Beh. 2012;17(6):581-589.

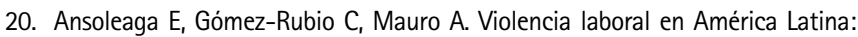
una revisión de la evidencia científica. Vertex. 2015;36:444- 452

21. Hernández $S$, Fernández $C$, Baptista P. Metodología de la Investigación. México: McGraw Hill; 2003.

22. Ferrerira I, Urrútia G, Alonso-Coello P. Revisiones sistemáticas y metaanálisis: bases conceptuales e interpretación. Rev Esp Cardiol [en línea]. 2011;64(8):688696 [consultado jun 2016]. Disponible en http://www.revespcardiol.org/es/ revisiones-sistematicas-metaanalisis-bases-conceptuales/articulo/90024424/

23. Andréu, J. Las técnicas de Análisis de Contenido: Una revisión actualizada [en línea]. sl; 2000 [consultado jun 2016]. Disponible en: public.centrodeestudiosandaluces.es/pdfs/S200103.pdf

24. Urrutia G, Bonfill X. Declaración PRISMA: una propuesta para mejorar la publicación de revisiones sistemáticas y metaanálisis. Med Clín. 2010;135 (11):507511.

25. SCImago Lab / CONICYT. Principales indicadores cienciométricos de la actividad científica chilena 2012. Informe 2014: una mirada a 10 años [en línea]. MadridValparaiso:CONICYT-SCImago Lab.; 2014 [consultado jun 2016]. Disponible en http://www.conicyt.cl/informacioncientifica/files/2014/09/IndicadoresCienciom\%C3\%A9tricos-2014.pdf

26. Fontes KB, Santana RG, Pelloso SM, Carvalho MDB.Factors associated with bullying at nurses' workplaces. Rev latino-am enfermagem. 2013; 21(3):758764.

27. Rodriguez N. Mobbing y nivel de estrés en el ámbito académico. Acta Psiquiatr Psicol Am Lat. 2010; 56(2):91-95.

28. Soares $L R$, Villela, WV. 0 assédio moral na perspectiva de bancários. Rev bras saúde ocup. 2012; 37(126):203-212.

29. Vasconcellos I de, Griep R, Lisboa M, Rotenberg L. Violence in daily hospital nursing work. Acta paul enferm. 2012; 25(No.spec. 2):40-47.

30. Batista C, Campos A, Reis J, Schall V. Violência no trabalho em saúde: análise em unidades básicas de saúde de Belo Horizonte, Minas Gerais. Trab educ saúde. 2011; 9(2): 295-317.
31. Rigotto $R$, Maciel R, Borsoi I. Produtividade, pressão e humilhação no trabalho: os trabalhadores e as novas fábricas de calçados no Ceará. Rev bras saúde ocup. 2010; 35(122):217-228.

32. Fidalgo A, Piñuel I. La escala CISNEROS como herramienta de valoración del mobbing. Psicothema. 2004;16(4):615 -624.

33. Pando $\mathrm{M}$, Bertot $\mathrm{M}$, Aranda-Beltrán $\mathrm{C}$, Amezcua $\mathrm{MT}$. Análisis factorial exploratorio del inventario de violencia y acoso psicológico en el trabajo (IVAPT) para Cuba. REMESAT. 2011;1(8):10-14.

34. González C, Pincheira P, Moyano E. Análisis de fiabilidad y estructura factorial del inventario de violencia y acoso psicológico en el trabajo IVAPTPANDO. En: Moyano E, editor. Calidad de vida y psicología en el Bicentenario de Chile. Santiago: Marmos; 2010. p. 507-537.

35. Pando $M$, Aranda $C$, Olivares D. Análisis factorial confirmatorio del inventario de violencia psicológica y acoso psicológico en el trabajo (IVAPTPANDO) para Bolivia y Ecuador. Liberabit. 2012; 18(1):27-36.

36. Pando $M$, Aranda $C$, Parra L, Gutiérrez AM. Determinación del mobbing y validación del Inventario de Violencia y Acoso Psicológico en el Trabajo (IVAPT) para Colombia. Salud Uninorte. 2013; 29(3):525-533.

37. Tovalin $H$, Rodriguez $M$, Unda $S$, Centeno J, Martinez $E$, Orduña J, Pérez R. Validación y comparación de dos instrumentos para detectar la presencia de hostigamiento psicológico en el trabajo [en línea]. 1er Congreso internacional y 4a Foro de Las Américas en la Investigación sobre Factores Psicosociales, estrés y Salud Mental en el trabajo; 17-20 oct. 2012; Bogotá, Colombia. Bogotá: Universidad de los Andes; 2012 [consultado jun 2016]. Disponible en: http://factorespsicosociales.com/primercongreso/pdfs/ Posters/Poster27.pdf

38. Meurer Antunes B, Sandra Carlotto M, Neves Strey M. Mulher e trabalho: visibilizando o tecido e a trama que engendram o assédio moral. Psicol rev (Belo Horizonte). 2012; 18(3):420-445.

39. Souza Junior, A, Cerquinh, K.G., Nogueira, R.J. Melo, D. R. Aspectos da dominação masculina no assédio moral ao profissional homossexual no polo industrial de manaus. Revista Pensamento \& Realidade. 2013; 28(1):83-103.

40. Fontes K, Santana R, Pellosos S, Carvalho M. Factors associated with bullying at nurses' workplaces. Rev. Latino-Am. Enfermagem. 2013; 21(3):758-764.

41. Silva E F, Oliveira, K K, Souza P C. Saúde mental do trabalhador: 0 assédio moral praticado contra trabalhadores com LER/DORT. Rev Bras Saúde Ocup. 2011;36(123):56-70

42. Acosta $M$, Aguilera $M$, Pozos B. Relación entre el acoso moral en el trabajo y el estilo interpersonal en el manejo de conflictos. Cienc Trab. 2009; 21:1417.

43. Jacoby $A R$, Falcke $D$, Lahm $C$, Nunes GAssédio moral: uma guerra invisivel no contexto empresarial. Revista Mal estar e Subjetividade. 2009; 9(2):619-645.

44. Rodrigues $M$, Freitas ME. Assédio moral nas instituições de ensino superior: um estudo sobre as condições organizacionais que favorecem sua ocorrência. Cad EBAPE BR. 2014; 12(2):284-301.

45. Lima CQ, Barbosa CM, Mendes RW, Patta CA. Assédio moral e violências no trabalho: caracterização em pericia judicial. Relato de experiência no setor bancário. Rev bras saúde ocup. 2014; 39(129):101-110.

46. Caran VC, Secco IA, Barbosa DA, Robazzi ML. Assédio moral entre docentes de instituição pública de ensino superior do Brasil. Acta paul enferm. 2010; 23(6):737-744.

47. Marques RD, Martins Filho E, Paula GS de, Santos ER dos. Assédio moral nas residências médica e não médica de um hospital de ensino. Rev bras educ med. 2012; 36(3):401-406. 